\title{
Topical ophthalmic amethocaine alleviates trigeminal neuralgia pain
}

\author{
This article was published in the following Dove Press journal: \\ Local and Regional Anesthesia \\ 10 December 2010 \\ Number of times this article has been viewed
}

\author{
Silviu Brill' \\ Ron Ben-Abraham' \\ Itay Goor-Aryeh ${ }^{2}$ \\ 'Pain Clinic, Tel-Aviv Medical Center, \\ Tel-Aviv, Israel; ${ }^{2}$ Pain Clinic Sheba \\ Medical Center, Tel-Hashomer, Israel
}

Background: Trigeminal neuralgia (TGN) is a challenging pain syndrome.

Aim: To test the effectiveness of local instillation of ophthalmic solution of amethocaine in relieving the pain of TGN.

Methods: We performed an open-label prospective study in 40 consecutive currently treated patients suffering from TGN with a reported visual analog scale (VAS) $>8$ (severe pain). The patients received two drops of amethocaine $1 \%$ instilled on the cornea ipsilateral to the painful side. Pain score assessment using VAS was recorded pre- and post-treatment.

Results: A total of $32(80 \%)$ patients reported a significant reduction in pain 10 minutes after drops instillation as compared with pre-treatment pain score. Pre-treatment VAS score was $8.53 \pm 0.6$ as compared with $4.78 \pm 1.83$ post-amethocaine treatments $(P<0.00001)$.

Conclusion: Topical ophthalmic instillation of amethocaine $1 \%$ can be considered as an immediate effective method for pain paroxysm of TGN.

Keywords: amethocaine, trigeminal neuralgia, pain, opthalmic

\section{Introduction}

Trigeminal neuralgia (TGN), also called "tic douloureux", ${ }^{1}$ is a neurological condition affecting the sensory division of the fifth cranial (trigeminal) nerve and characterized by recurrent episodes of severe, shock-like pain confined to the distribution of one or more of the nerve's three major branches: the ophthalmic (V1), maxillary (V2), or mandibular (V3). Typically, excruciating, lancinating facial pain occurs following stimulation of specific trigger zones by movement or touch. The paroxysmal pain may be felt in the lips, gums, cheek, or chin. ${ }^{2}$ TGN occurs in about 1 in 25,000 in the general population and is uncommon before the third decade of age.

Pharmacotherapy is at present the first-line treatment for TGN and includes analgesics, anticonvulsants, and antidepressants. Surgical treatment is reserved for refractory and long-lasting pain.

Amethocaine, a 4-(butylamino)benzoic acid 2-(dimethylamino)ethyl ester, is a potent surface local anesthetic that acts by blocking sensory nerve endings near the site of application. In ophthalmology, amethocaine hydrochloride is used to anesthetize the cornea during ophthalmological procedures. When tested before, in a double-blind, placebo-controlled study, Kondziolka et al found that a single application of an eye drop of amethocaine did not cause any statistically significant pain relief in patients with classical TGN. ${ }^{3}$

The aim of the present study was to further test the effectiveness of local instillation of ophthalmic solution of amethocaine $1 \%$ in relieving the pain of TGN. 


\section{Material and methods}

A total of 40 consecutive patients (M-17, F-23) previously diagnosed with TGN and attending our pain clinic from January 1, 2005 to June 1, 2007 were included in this prospective case study. The diagnosis was made according to International Association for the Study of Pain (IASP) criteria.

\section{Instillation method}

Two drops of amethocaine $1 \%$ were instilled onto the cornea ipsilateral to the painful side. After instillation the eye was closed for 10 minutes and the patient remained for surveillance in the pain clinic for another 20 minutes. For the patients with no significant pain reduction a second eye instillation was performed 15 minutes after the initial instillation. Changes in the severity of pain and frequency of attacks were assessed using the student $t$-test for paired data.

\section{Results}

When attending the pain clinic, all patients suffered from severe typical sharp and lancinating pain in one or more trigeminal nerve branch distributions, facial hyperesthesia, and a visual analog scale (VAS) score $>8$ (Table 1). The pain attacks occurred despite the fact that patients continued their previous medications, which included nonsteroidal antiinflammatory agents, opioids, carbamazepine, amitriptyline, duloxetine, citalopram, gabapentin, and pregabalin.

The pain was either spontaneous or aggravated by a trigger factor. Sensory branches of the trigeminal nerve that were affected included V1 (23), V2 (26), and V3 (23). Pain distribution in two divisions of the nerve occurred in 16 cases, and in all three divisions in eight cases.

A total of $32(80 \%)$ patients reported a significant reduction in pain 10 minutes after instillation of the drops as compared with pre-treatment pain score. Pre-treatment VAS score was $8.48 \pm 0.64$ as compared with $4.53 \pm 1.77$ post-amethocaine treatments $(P<0.0001)$ (Table1). The pain reduction was noted regardless of the trigeminal distribution of the symptoms. All the patients also reported a reduction in the frequency of attacks for the following 24 hours (reported by pain diary). There were no adverse events reported in our patients.

Table I Patient demographics and pain reduction (mean \pm SD)

\begin{tabular}{lllll}
\hline $\begin{array}{l}\text { Patient } \\
\text { (N) }\end{array}$ & $\begin{array}{l}\text { Age } \\
\text { (years) }\end{array}$ & $\begin{array}{l}\text { Pain } \\
\text { duration } \\
\text { (years) }\end{array}$ & $\begin{array}{l}\text { Pain intensity } \\
\text { (VAS score) }\end{array}$ \\
\hline 40 & & Pre & Post \\
\hline
\end{tabular}

Note: $* P<0.0001$.

Abbreviations: SD, standard deviation; VAS, visual analog scale.

\section{Discussion}

The first description of TGN was attributed to John Fothergill in 1773 (Fothergill's disease). However, early descriptions can be found in Avicenna's writings in the 11 th century. The International Association of Pain defines TGN as a recurrent, usually unilateral, brief stabbing pain in the distribution of one or more branches of the fifth cranial nerve. ${ }^{2}$

Amethocaine hydrochloride is a local anesthetic used for minor conjunctival and corneal surgery and is especially useful for the alleviation of pain resulting from removal of foreign bodies from the eye. Our study demonstrated the effectiveness of the application of amethocaine ophthalmic drops in offering a quick relief of the excruciating pain of TGN attacks.

TGN may be idiopathic (classic) or secondary to multiple sclerosis or benign compression of the Gasserian ganglion (the sensory ganglion of the trigeminal nerve). ${ }^{4}$ The sensory root of the trigeminal nerve expands into the trigeminal ganglion, which contains the cells of origin of the sensory fibers and from which the three divisions of the nerve arise; it supplies the face, teeth, mouth, and nasal cavity. In 1991, Zavon and Fichte discovered the possibility of using eye anesthetics (proparacaine) for TGN by accident. Zavon, who suffered from TGN, found that his pain vanished for over a year immediately after an eye anesthetic was applied (for corneal ulceration). They then tested the anesthetic on another TGN patient and achieved pain relief for over a month. ${ }^{5} \mathrm{We}$ should note that repeated use of eye anesthetics may cause toxic keratopathy and may also slow down the healing of eye wounds. Hence, it was recommended that eye anesthetics must be used with great care and that patients should be advised not to rub the eye for at least an hour. However, in our patients, we have not noticed any adverse effects.

Although the pathophysiology and precise etiology of TGN are unknown, both central and peripheral mechanisms have been proposed. Current evidence suggests focal demyelination as a result of vascular compression of the central axons of the trigeminal nerve to be the underlying cause of TGN. ${ }^{4}$ It therefore seems that there are several possible theoretical explanations for the beneficial effect of amethocaine ophthalmic drops in relieving TGN pain attacks, eg, 1) peripheral (corneal) suppression of the trigger stimulus might influence the central perception of pain in all three divisions of the trigeminal nerve; 2) migration of local anesthetic from the cornea to the sphenopalatine ganglion; and 3) a central mechanism, with retrograde transport of local anesthetic to the Gasserian ganglion.

According to one suggested theory, the explanation is that peripheral injury or disease of the trigeminal nerve increases 
afferent firing in the nerve perhaps by ephaptic transmission between afferent unmyelinated axons and partially damaged myelinated axons, and the amethocaine may cease this aberrant activity.

Treatment objectives in the case of TGN are to eliminate pain, reduce the likelihood of recurrence, and prolong the time to recurrence by selectively destroying pain fibers without inducing excessive sensory loss, motor dysfunction, or other complications. Surgical treatment, including microvascular decompression; partial trigeminal rhizotomy; stereotactic radiosurgery; radiofrequency denervation; cryotherapy; or chemical denervation targeted to the root (rhizotomy), ganglion (gangliolysis), or branches of the trigeminal nerve, were suggested as potential means of treatment of TGN. ${ }^{6,7}$ However, these involve invasive procedures and substantial risk of complication as well as the need for extra expertise. Peripheral nerve blocks with high concentrations of local anesthetics were previously proved to be safe and useful in the treatment of TGN, leading to pain relief for weeks after an infraorbital block..$^{8,9}$ The treatment of TGN in general is hampered by poor-quality methodological trials.

Our experience presents the potential beneficial effect of topical ophthalmic amethocaine in alleviating TGN pain attacks. Due to the simplicity of this treatment, we suggest that it be considered as a first-line option to use for severe pain attacks in TGN. As the pain in TGN tends to be paroxysmal, treatment with ophthalmic amethocaine may be recommended as a rapid and effective therapeutic measure, especially when the patient cannot attend the pain clinic or is at home. Nevertheless, a prudent approach justifies more extensive randomized placebo-controlled trials with this treatment in order to further explore ideal dosage and possible side effects.

\section{Disclosure}

No conflicts of interest were declared in relation to this paper.

\section{References}

1. Cole CD, Liu JK, Apfelbaum RI. Historical perspectives on the diagnosis and treatment of trigeminal neuralgia. Neurosurg Focus. 2005; 15:18:E4

2. Kitt CA, Gruber K, Davis M, Woolf CJ, Levine JD. Trigeminal neuralgia: opportunities for research and treatment. Pain. 2000;85:3-7.

3. Kondziolka D, Lemley T, Kestle JR, et al. The effect of single-application topical ophthalmic anesthesia in patients with trigeminal neuralgia. A randomized double-blind placebo-controlled trial. J Neurosurg. 1994;80:993-997.

4. Nurmikko TJ, Eldridge PR. Trigeminal neuralgia: pathophysiology, diagnosis and current treatment. Br J Anaesth. 2001;87:117-132.

5. Zavon MR, Fichte CM. Trigeminal neuralgia relieved by ophthalmic anesthetic. JAMA. 1991;265:2807.

6. Lopez BC, Hamlyn PJ, Zakrzewska JM. Systematic review of ablative neurosurgical techniques for the treatment of trigeminal neuralgia. Neurosurgery. 2004;54:973-982.

7. Mathews ES, Scrivani SJ. Percutaneous stereotactic radiofrequency thermal rhizotomy for the treatment of trigeminal neuralgia. Mt Sinai J Med. 2000;67:288-299.

8. Radwan IA, Saito S, Goto F. High-concentration tetracaine for the management of trigeminal neuralgia: quantitative assessment of sensory function after peripheral nerve block. Clin J Pain. 2001;17:323-326.

9. Goto F, Ishizaki K, Yoshikawa D, Obata H, Arii H, et al. The long lasting effects of peripheral nerve blocks for trigeminal neuralgia using high concentration of tetracaine dissolved in bupivacaine. Pain 1999;79:101-103.

\section{Publish your work in this journal}

Local and Regional Anesthesia is an international, peer-reviewed, open access journal publishing on the development, pharmacology, delivery and targeting and clinical use of local and regional anesthetics and analgesics. The journal welcomes submitted papers covering original research, basic science, clinical studies, reviews \& evaluations, guidelines, expert opinion and commentary, case reports and extended reports. The manuscript management system is completely online and includes a very quick and fair peer-review system, which is all easy to use. Visit http://www.dovepress.com/testimonials.php to read real quotes from published authors. 